pendent of both its ATPase activity and its ability to dissociate SNARE complexes (Fig. 1d). The authors study in vitro membrane fusion that can reform Golgi cisternae. During mitosis, the Golgi apparatus within the cell is fragmented into smaller vesicles that can be distributed between the daughter cells. Subsequently, these postmitotic Golgi fragments fuse with one another to reform the Golgi's proper cisternae. This fusion can be reconstituted in vitro and depends on NSF and ATP. Müller et al. test the fusing abilities of an engineered NSF mutant (in which amino-acid residue glycine 274 is mutated to glutamate), which is based on a temperature-sensitive allele of Drosophila NSF called comatose ${ }^{17}$; flies with this allele are paralysed at raised temperatures ${ }^{8}$. As Müller et al. hoped, the mammalian NSF bearing the same mutation is also temperature sensitive and promotes cisternal regrowth at $25^{\circ} \mathrm{C}$, but not at $37^{\circ} \mathrm{C}$. But the surprise lies elsewhere: even at the permissive temperature of $25^{\circ} \mathrm{C}$, this mutated NSF seems to lack detectable ATPase activity and does not dissociate SNARE complexes (though it can still bind the SNAREs). The authors bolster their claim that fusion in this assay does not require ATPase activity by showing that a mutant with an independent mutation lying in the catalytic site of the D1 ATPase also retains the ability to promote Golgi membrane fusions. How then do the mutants promote fusion?
One feature of the paper that may receive close scrutiny is the relative fusion potency of the mutant and wild-type NSFs. The assay is conducted with high concentrations of NSF, where a 100 -fold dilution of the wild-type enzyme produces only a $30 \%$ decrease in cisternal regrowth (see Fig. 1 on p. 336). At these concentrations, the mutant NSF (which is not as potent as the wild type on a per microgram basis) might be getting by with a small amount of residual ATPase. But the authors argue strongly that they found no residual ATPase and SNARE-dissociating activity in this mutant.

Fusion without ATP? This is not per se a shock. Physiologists studying endocrine cells and nerve terminals have long known that the fusion of secretory vesicles with the plasma membrane (exocytosis) can proceed after all the ATP has been dialysed from a cell and in the presence of chelators that would remove any free $\mathrm{Mg}^{2+}$. Thus the final, $\mathrm{Ca}^{2+}$-triggered step of exocytosis cannot be ATPase dependent. On this basis, NSF at the synapse has been relegated to a priming role such as those discussed above. But if there are systems in which NSF has functions besides being an ATPase, NSF could be moved back into the spotlight for the final act of fusion.

Müller et al.'s is the second NSF paper of the year to undermine our confidence in the centrality of SNARE dissociation for NSF activity. Otter-Nilsson et al. ${ }^{9}$ have reported a reconstituted fusion assay for lipid vesicles (liposomes) in which NSF and its kindred protein $\mathrm{p} 97$ drove membrane fusion in the absence of the SNARE proteins (Fig. 1c). The efficacy of p97 and NSF in this minimal reconstituted system was impressive. $\mathrm{p} 47$, a protein related to $\alpha$-SNAP, enhanced NSF performance in the assay. If this reconstituted system at all resembles in vivo membrane fusion, the implication would be that NSF-like proteins can directly mediate the fusion of membranes and that, though SNAREs may help by recruiting the NSF to the membrane, it is the NSF that promotes membrane mixing. This bilayer-fusing function for NSF could be precisely what is effective in the Golgi-reassembly assay of Müller et al. - an NSF function that does not require the NSF to dissociate SNAREs. However, there is an inconsistency to be resolved: the fusion activity observed in liposomes required ATP and non-hydrolysable ATP- $\gamma S$ did not work ${ }^{9}$, but in the Golgireassembly assay the ATPase activity of NSF was dispensable ${ }^{3}$.

The study of membrane fusion has had more than its share of confusions and delusions and Müller et al. will probably draw fire for their unsettling result. Others will certainly wish to duplicate these results and probe for any residual ATPase activity that could reconcile the old model with the trafficking competence of these mutants. Not surprisingly, questions remain. Are the D1

\title{
Putting actin organization on a pedestal
}

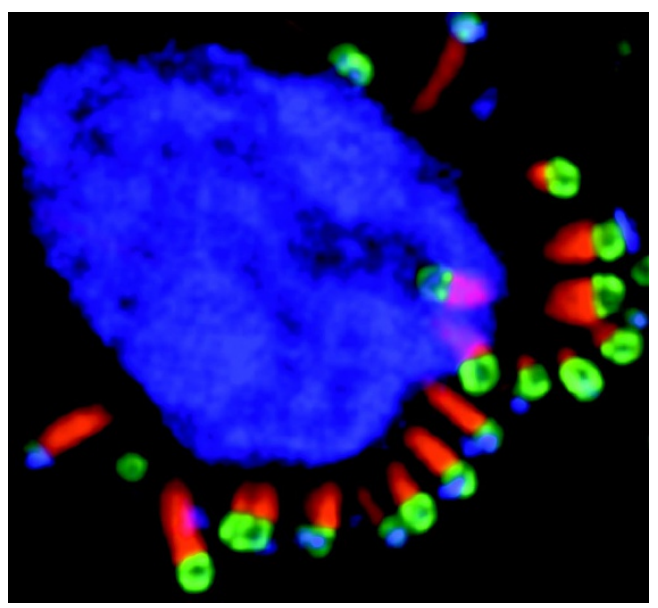

Like many other pathogens, enteropathogenic Escherichia coli (EPEC), a diarrhoea-causing bacterium responsible for the death of millions in developing countries, has evolved some cunning tricks for infecting host cells: it subverts normal cellular processes for its own ends. Attempts to understand this molecular trickery shed light on not only the pathogenic processes of EPEC infection but also the underlying cellular machinery. EPEC, which infects intestinal cells, binds to the surface of the cells and triggers the highly localized reorganization of the underlying cytoskeleton to form actin-rich pedestals thought to be crucial for EPEC infection. This picture depicts these pedestals in a HeLa cell exposed to EPEC, with the bacteria pseudocoloured in green, actin in red and bacterial and cellular DNA in blue. Elsewhere in this issue (Nature Cell Biol. 1, 389$391 ; 1999)$, Daniel Kalman and colleagues provide the first insights into the cellular mechanisms used by EPEC to induce pedestals, and identify at least two of the host factors involved.

They discover that cellular WASP proteins, known to be involved in actin reorganization in other systems, are specifically localized to the sites of EPEC attachment and that this is absolutely necessary to induce actin pedestals. WASP recruits the so-called Arp2/3 complex and stimulates its actin-polymerization activity, ultimately resulting in pedestal formation. So there are striking similarities and differences between how EPEC and some intracellular pathogens, such as Listeria monocytogenes and Shigella flexneri, activate actin polymerization.

But how does EPEC recruit WASP? Interestingly, the authors show that a domain of WASP that can bind members of the small-GTPase family is both required and sufficient for WASP localization to the sites of EPEC attachment, indicating that an as-yet-unknown GTPase may be involved in this process. They cite preliminary evidence suggesting that a recently identified cellular GTPase named Chp may be the culprit. Identifying this cellular GTPase, as well as other factors that link the bacteria to the host-cell machinery, will be exciting. We are certain to learn a lot more in the future about the interesting molecular devices used by EPEC to infect its host cells, and, more fundamentally, about the control of the actin cytoskeleton itself.

BARBARA MARTE 